\title{
Concepções de estudantes universitários brasileiros sobre os fatores e as funções da aprendizagem
}

\author{
Luiz Gustavo Lima Freire ${ }^{a}$ \\ António Manuel Duarte ${ }^{b}$
}

\section{Resumo}

Este artigo expõe parte dos resultados de uma investigação que teve como objetivo explorar as concepções de aprendizagem de um grupo de estudantes universitários brasileiros, à luz da perspectiva fenomenográfica: os resultados relativos às suas concepções sobre os fatores e as funções de aprendizagem. Quinze estudantes de um curso de Psicologia foram entrevistados com base num guião de uma entrevista semiestruturada. As respostas, sujeitas a uma análise de conteúdo, revelaram a atribuição, à aprendizagem, de funções adaptativas, cognitivas, motivacionais e interpessoais, assim como de fatores pessoais e ambientais. Os resultados permitem a constatação de uma replicação de parte das concepções sobre os fatores e as funções da aprendizagem anteriormente reveladas por outros estudos fenomenográficos, assim como do surgimento de concepções aparentemente novas.

Palavras-chave: Aprendizagem acadêmica. Concepções de aprendizagem. Fenomenografia.

\section{Introdução}

$\mathrm{Na}$ área da investigação psicológica sobre a aprendizagem acadêmica, destaca-se, entre outras, a perspectiva teórica fenomenográfica, centrada nas concepções (nas noções ou significados) dos indivíduos sobre o fenômeno da aprendizagem (KHAN, 2014; MARTON; DALL'ALBA; BEATY, 1993) ${ }^{1}$. A fenomenografia realça

\footnotetext{
a Faculdade dos Guararapes Laureate International Universities. Jabotão dos Guararapes, Pernambuco, Brasil.

b Universidade de Lisboa, Faculdade de Psicologia. Lisboa, Portugal.

1 A palavra Fenomenografia possui duas raízes: fenômeno, que significa tudo quanto é percebido pelos sentidos ou pela consciência, e grafia, que se refere à representação ou esquema. A sua origem etimológica vem do grego pháinomenon, ou aparência, e gráphein, ou descrição, logo, descrição das coisas como elas se apresentam aos indivíduos (MARTON; FAI, 1999).
} 
o fato de que a concepção que os estudantes têm da aprendizagem se relaciona com outras das suas características pessoais (cognitivas, afetivas e interpessoais), e com uma diversidade de aspectos ambientais (objetivos educativos, conteúdos, métodos, materiais e recursos educacionais). Em especial, ela está em estreita relação com as suas "abordagens à aprendizagem", definidas em termos do tipo de motivação e de estratégias que apresentam face às tarefas (ASIKAINEN, 2014; BIGGS, 1987; LARA et al., 2011; LEUNG; WONG; WONG, 2013; MARTON; SÄLJÖ, 1976; YANG; TSAI, 2010). Efetivamente, tal como referido por Marton e Booth (1997, p. 111):

[...] in order to make sense of how people handle problems, situations, the world, we have to understand the way in which they experience the problems, the situations, the word, that they are handling or in relation to which they are acting. Accordingly, a capability for acting in a certain way reflects a capability experimenting something in a certain way.

A este propósito, note-se que a perspectiva fenomenográfica tem origem num estudo de Marton e Säljö (1976) em que se pediu a estudantes que lessem um artigo acadêmico para posteriormente responderem a questões sobre como o haviam lido. As respostas demonstraram que, para aquela leitura, os estudantes se relacionavam com a tarefa basicamente de duas formas: através de uma abordagem dita "de superfície", que consistia numa leitura mais "mecânica", com ênfase na memorização das palavras e do formato do texto ou, em alternativa, através de uma abordagem dita "de profundidade", sobretudo baseada na compreensão do significado do texto. Mais tarde, outros estudos identificaram, ainda, a existência de um terceiro tipo de abordagem, denominada "estratégica" ou "de sucesso", orientada para as classificações através de uma estratégia de organização pessoal (BIGGS; MOORE, 1993).

A fenomenografia defende assim, que, para compreender a aprendizagem de forma abrangente, é necessário entendê-la a partir da perspectiva do próprio estudante. Deste modo, e seguindo uma linha construtivista, a fenomenografia realça que os estudantes se comportam não apenas em função dos dados "objetivos" característicos do processo de aprendizagem mas, igualmente, em função da forma como concebem essa mesma aprendizagem.

Neste sentido, o estudo sobre as concepções de aprendizagem dos estudantes é relevante, não apenas para a compreensão do insucesso acadêmico, mas também, para que, através de intervenções dirigidas à mudança dessas concepções, possa-se melhorar a qualidade da aprendizagem (BIGGS, 2010; 1990; MARTON, 1988; SÄLJÖ, 1984). 
Paralelamente, como defende Entwistle (1984), na medida em que os professores compreendam as concepções dos seus estudantes, poderão mais facilmente incentivar a utilização de estratégias de aprendizagem mais adaptadas. Mais, uma das condições para que os professores possam refletir sobre a forma como ensinam consiste em basearem o seu pensamento no que se sabe sobre como os estudantes aprendem (BIGGS, 2010).

A investigação fenomenográfica envolve, sobretudo, a utilização de métodos qualitativos de pesquisa recorrendo por excelência à entrevista e à análise de conteúdo, sendo as diferentes formas de concepção expressas por meio de categorias que captam pontos de vista diversificados sobre o aprender. Estas concepções não são tidas como completas ou finais, porque a experiência e a consciência não são exaustivas, mas dirigidas pelos objetivos e enfoques pessoais (MARTON, 1981).

Para além disso, a investigação fenomenográfica tem vindo a estudar as concepções de aprendizagem considerando diferentes dimensões deste fenômeno, tendo vindo a centrar-se, a maior parte dos estudos, na dimensão referencial (noção do que é a aprendizagem) na dimensão processual (noção de como se aprende) e na dimensão contextual (noção de onde se aprende) (MARTON; DALL'ALBA; BEATY, 1993). Considerando estas dimensões principais, tem-se constatado a existência de duas formas básicas dos estudantes conceberem a aprendizagem: segundo uma concepção "quantitativa" ou "qualitativa" (ASIKAINEN, 2014; BIGGS; MOORE, 1993; KHAN, 2014; LARA et al., 2011; MARTON; BOOTH, 1997; MARTON; SÄLJÖ, 1977; MARTON; DALL'ALBA; BEATY, 1993; YANG; TSAI, 2010)².

Em termos gerais, a concepção "quantitativa" de aprendizagem vê esta como acumulação de informação, através da memorização, no contexto educacional. Por outro lado, a concepção "qualitativa" de aprendizagem toma esta como compreensão de conhecimento, por meio da análise e relacionação da informação, assim como de reinterpretação e mudança pessoal, no contexto amplo do ambiente. Esta dicotomia de concepções de aprendizagem tem sido replicada em diferentes países, incluindo o caso de Portugal (DUARTE, 2007). Note-se, no entanto, a existência de um elemento comum às duas representações gerais da aprendizagem - a noção de que esta é uma forma de aumentar a capacidade de conhecer, pensar ou realizar alguma coisa através da experiência (PRAMLING, 1986). De acordo com Marton, Dall'Alba e Beaty (1993), outro elemento comum é o da existência de uma fase de aquisição e de uma fase de aplicação do que é aprendido, o que naturalmente faz emergir a ideia de que a aprendizagem se desenvolve em torno de um eixo temporal.

Esta diferenciação tem eco na classificação de Paulo Freire (2003) da Pedagogia como "bancária" ou "problematizadora". 
Por outro lado, sobretudo em estudos mais recentes, tem surgido uma atenção a outras dimensões das concepções de aprendizagem, para além das três básicas atrás referidas, que nomeadamente consideram a noção dos estudantes sobre os fatores (dimensão fatorial) e as funções da aprendizagem (dimensão funcional). Num estudo com estudantes do $5^{\circ}$ ano de escolaridade, Rosário et al. (2006) identificaram uma diversidade de concepções sobre a função da aprendizagem: para "garantir" o futuro, ajudar as outras pessoas, aumentar os conhecimentos, defender-se, estar com as pessoas, interagir na sociedade, realizar-se pessoalmente, resolver os problemas, reagir às situações e aplicar o aprendizado na prática do dia a dia. Em um outro estudo, com estudantes do Ensino Secundário, Rendeiro (2005) constatou que aqueles podem conceber a aprendizagem para a avaliação como dependente, quer de fatores pessoais (cognitivos, afetivos, orgânicos, interpessoais) quer de fatores ambientais (contexto de ensino e de avaliação) quer da sorte. Rebelo (2005), por sua vez, constatou que os estudantes do Ensino Superior podem atribuir uma diversidade de funções à aprendizagem com o computador, tais como adquirir informação, diversificar o modo de aprender, facilitar a aprendizagem através da comunicação, construir e transferir conhecimento, facilitar ou desenvolver competências de aprendizagem cognitivas e de produção do discurso, automatizar a aprendizagem ou fortalecer a autonomia e motivar para a aprendizagem.

Embora a investigação fenomenográfica sobre as concepções que os indivíduos têm da aprendizagem esteja bem desenvolvida (desde a década de setenta do século passado), escasseia no Brasil a investigação sobre esta variável, tal como acontece para a variável da abordagem à aprendizagem, como apontado por Litto (2015). Por outro lado, a maior parte dos estudos fenomenográficos tem incidido, sobretudo na dimensão referencial, processual e contextual daquela concepção (a noção sobre a natureza, o processo e o contexto da aprendizagem), negligenciando outras dimensões potencialmente relevantes. Constata-se, assim, a necessidade, quer de investigar as concepções dos indivíduos sobre a aprendizagem no contexto nacional brasileiro, quer de desenvolver este tipo de investigação considerando outras dimensões da mesma variável.

Na sequência de um estudo anterior (FREIRE; DUARTE, 2010), que explorou as concepções de estudantes universitários brasileiros sobre a natureza, o processo e o contexto da aprendizagem, o objetivo deste estudo é o de explorar a variação qualitativa da concepção que os mesmos estudantes têm sobre os fatores e as funções da aprendizagem. O estudo orienta-se assim, complementarmente, para duas dimensões da concepção de aprendizagem, a funcional e a fatorial, pouco exploradas na literatura. Para além de ajudar a colmatar a aparente escassez de conhecimento sobre as concepções de aprendizagem dos estudantes brasileiros, este estudo pretende assim contribuir para a expansão do conhecimento sobre dimensões menos exploradas das concepções. 


\section{Método}

Para atingir o objetivo proposto, de explorar a variação qualitativa da concepção que os estudantes têm sobre os fatores e as funções da aprendizagem, foi realizado um estudo qualitativo que envolveu a realização de entrevistas e de uma análise de conteúdo das respostas.

A amostra de participantes entrevistados, constituída por conveniência, compreendeu 15 indivíduos que faziam parte da população dos estudantes que frequentavam o primeiro e segundo períodos de um curso superior de Psicologia de uma faculdade particular da região metropolitana da cidade do Recife, no Brasil. A idade dos participantes variava entre os 17 e os 25 anos, com uma média de 20,3 anos, sendo que 14 eram do sexo feminino e um do sexo masculino. O número de participantes não foi pré-determinado, tendo resultado da aplicação do critério de "saturação" das categorias reveladas pela análise de conteúdo a que as entrevistas foram sujeitas. Ou seja, foram sendo realizadas entrevistas até que a inclusão de novos participantes pareceu não trazer respostas qualitativamente diferentes das fornecidas nas entrevistas anteriores. A entrevista, de tipo semiestruturada, foi realizada com base num guião com as seguintes questões abertas: 1 . O que é aprender? (dimensão referencial) 2. Como se aprende? (dimensão processual) 3. Onde se aprende? (dimensão contextual) 4. Quais são os fatores que influenciam a aprendizagem? (dimensão fatorial) 5. Qual é a função da aprendizagem? (dimensão funcional). Como já referido, neste artigo serão apresentados apenas os resultados relativos à dimensão funcional e a fatorial. Após aprovação do estudo pelo Comitê de Ética em Pesquisa da Faculdade onde foi implementado, procedeu-se à realização das entrevistas. Cada entrevistado forneceu o seu consentimento, depois de ter sido apresentado aos objetivos e metodologia do estudo, à forma de participação voluntária e à confidencialidade dos dados. As entrevistas foram gravadas em áudio e posteriormente transcritas. Os estudantes foram informados que, ao responderem às questões da entrevista, não se deveriam centrar na sua forma particular de aprender, mas na aprendizagem em geral.

A análise das respostas foi efetuada mediante análise de conteúdo, que envolveu três momentos principais: 1) segmentação das unidades a categorizar; 2) desenvolvimento de um sistema de categorias de análise; 3) testagem de confiança das categorias detectadas por comparação entre juízes. O critério utilizado para a segmentação foi o de "unidades temáticas" proposto por Flores (1994), ou seja, todo o segmento de resposta que abordasse, pelo seu conteúdo, qualquer das dimensões focadas do fenômeno da aprendizagem era considerado 
como uma unidade a categorizar, por correspondência com uma das categorias da respetiva dimensão do sistema (em desenvolvimento).

Considerando a crítica ao método "fenomenográfico", de que este aliena os participantes dos seus depoimentos, ao isolar e retirar frases soltas do contexto em que estas foram produzidas (SÄLJÖ, 1984), a categorização procurou ser sensível à totalidade de cada resposta, de acordo com a sugestão de Svensson (1989 apud EKLUND-MYRSKOG, 1998). Não foram consideradas respostas em que os indivíduos se referiam à sua aprendizagem pessoal, pois não se pretendia investigar a forma particular dos estudantes aprenderem, mas o que eles consideravam sobre a aprendizagem no geral.

Para a categorização das unidades segmentadas, considerando as dimensões da concepção de aprendizagem focalizadas neste artigo (dimensão "funcional" e dimensão "fatorial"), desenvolveu-se um sistema de categorias "indutivamente", ou seja, sem partir de categorias pré-existentes. Com base nos resultados dessa primeira etapa, foi possível chegar a um segundo sistema de categorias de descrição das concepções sobre os fatores e funções da aprendizagem, que foi depois utilizado numa segunda análise das respostas por dois juízes.

Numa "fase de formação dos juízes", estes procederam à leitura e discussão interpretativa do segundo sistema de categorias. Seguidamente, cada um realizou, de forma individual, independente, a categorização de cerca de $20 \%$ das respostas. Depois, com base em tabelas de contingência, foi estudado o padrão de acordo/desacordo entre eles. Atendendo à detecção, por um dos juízes, de categorias não previstas no segundo sistema de categorização, foi posteriormente construído um terceiro e último sistema, com base no qual cada um procedeu à categorização independente de todas as respostas. A magnitude de acordo entre os juízes foi de $77 \%$ para a dimensão fatorial e de $89 \%$ para a dimensão funcional. Para todos os cálculos de coeficiente de acordo foi utilizada a fórmula sugerida por Bakeman e Gottman (1997): $\mathrm{PA}=(\mathrm{Na} /(\mathrm{Na}+\mathrm{Nd})) 100$; em que "PA" é percentagem de acordo, "Na" é frequência de acordo e "Nd" é a frequência dos desacordos.

Finalmente, foi calculada a frequência relativa (\%) das categorias encontradas para cada dimensão (nas entrevistas em que a mesma categoria apareceu mais do que uma vez foi contabilizada apenas uma ocorrência). 


\section{Resultados}

A análise de conteúdo das respostas permitiu detectar a presença de nove categorias relativas à dimensão fatorial da concepção de aprendizagem. Cada categoria (e, nos casos em que se aplica, cada subcategoria) é ilustrada com um exemplo de uma resposta típica fornecida nas entrevistas (entre aspas, em itálico). As respostas fornecidas pelos estudantes exprimiram a ideia de que a aprendizagem é influenciada pelos seguintes fatores:

1. Fator Físico: ambientes, contextos, espaço e tempo físicos - "o ambiente influencia o processo de aprendizagem".

2. Fator Material: recursos materiais, objetos, artefatos - "materiais como livros, fonte como televisão, computador".

3. Fator Social: núcleo social e meios sociais e culturais - "influência social, cultural".

4. Fator Acadêmico: método de ensino e conteúdos programáticos - "[...] se o professor da universidade, manda você buscar, fazer pesquisas, desenvolver trabalho [...] curriculares, tudo isso influencia [...]".

5. Fator Cognitivo: raciocínio - "a partir do momento que eu [...] tenho [...] demora de raciocinar [...] vou tirando bem pouco [...] daquilo que eu vou aprender"; atenção - "é você tá observando, você tá prestando atenção".

6. Fator Afetivo: emoções - "o emocional, se você não tá bem emocionalmente [...] acho que você não consegue aprender"; persistência - "é necessário [...] uma insistência naquele comportamento, pra que haja a aprendizagem"; interesse - "o fato de como as pessoas valorizam, influencia".

7. Fator Interpessoal: convivência estabelecida com os demais - "a convivência, os relacionamentos sociais, as pessoas".

8. Fator Comportamental: formas de proceder - "o comportamento, a ação, forma de agir".

9. Fator Biológico: faixa etária, grau de maturação física e psicológica - "a maturidade de cada um vai constituindo a aprendizagem". 
No Quadro 1, pode-se consultar a frequência relativa (\%) de cada categoria, subcategoria e meta-categoria (agrupamento de categorias) relativas à dimensão fatorial da concepção de aprendizagem.

Quadro 1. Concepções dos fatores da aprendizagem.

\begin{tabular}{lccccc}
\hline Meta-Categoria & $\%$ & Categoria & $\%$ & Subcategoria & $\%$ \\
\hline \multirow{4}{*}{ Ambiental } & Físico & 26,6 & - & - \\
& \multirow{4}{*}{53,3} & Material & 6,6 & - & - \\
& & Social & 46,6 & - & - \\
& & Acadêmico & 20,0 & - & - \\
& & Cognitivo & 40,0 & Raciocínio & 13,3 \\
& & & Atenção & 40,0 \\
& & & Emoções & 33,3 \\
Pessoal & \multirow{2}{*}{ Afetivo } & 80,0 & Persistência & 26,6 \\
& \multirow{2}{*}{100} & & Interesse & 46,6 \\
& & Interpessoal & 26,6 & - & - \\
& & Comportamental & 6,6 & - & - \\
& & Biológico & 6,6 & - & - \\
\hline
\end{tabular}

Fonte: dados da pesquisa (2015).

Relativamente à dimensão funcional da concepção de aprendizagem, observou-se a presença de três categorias de respostas. Segundo os estudantes entrevistados, a aprendizagem possui as seguintes funções:

1. Função Interpessoal: partilhar conhecimento - "pra transmitir pras outras pessoas"; interagir - "é bom você ter uma aprendizagem, vocêsaber [...] se [não] fica muito chata a situação, você fica muito constrangido de tá no meio, eu não vou sair com aquele pessoal, aquele pessoal [...] não vai ter espaço pra mim”.

2. Função Motivacional: profissionalizar-se - "fazer um concurso público"; realizar-se - "ser melhor como pessoa"; concorrer com outras pessoas - "quando você bota numa balança [...] quem tem mais aprendizagem é que vence"; obter reconhecimento social - "se eu não for ninguém, eu vou ser julgado".

3. Função Cognitiva: conhecer - "para ter um esclarecimento maior, pra desenvolver os seus problemas, organizar suas ideias"; atualizar-se - "estar bem atualizado, tá sempre aprendendo, sempre renovando"; 
4. Função Adaptativa: viver - "pra viver"; garantir uma vida confortável - "o futuro da pessoa depende da aprendizagem"; reagir adaptativamente - "pra que a gente possa aprender a reagir em situações semelhantes de formas diferentes"; autonomizar-se - "pra sua independência".

No Quadro 2, pode-se consultar a frequência relativa (\%) de cada categoria, subcategoria e meta-categoria (agrupamento de categorias) relativas à dimensão funcional da concepção de aprendizagem.

Quadro 2. Concepções das funções da aprendizagem.

\begin{tabular}{|c|c|c|c|c|c|}
\hline Meta-categoria & $\%$ & Categoria & $\%$ & Subcategoria & $\%$ \\
\hline \multirow{2}{*}{ Interpessoal } & \multirow{2}{*}{40,0} & \multirow{2}{*}{ Interpessoal } & \multirow{2}{*}{40} & Partilhar conhecimento & 13,3 \\
\hline & & & & Interagir & 33,3 \\
\hline \multirow{10}{*}{ Intrapessoal } & \multirow{10}{*}{86,6} & \multirow{4}{*}{ Motivacional } & \multirow{4}{*}{66.6} & Profissionalizar-se & 46,6 \\
\hline & & & & Realizar-se & 46,6 \\
\hline & & & & Concorrer & 13,3 \\
\hline & & & & Obter Reconhecimento & 6,6 \\
\hline & & \multirow{2}{*}{ Cognitiva } & \multirow{2}{*}{53,3} & Conhecer & 46,6 \\
\hline & & & & Atualizar-se & 13,3 \\
\hline & & \multirow{4}{*}{ Adaptativa } & \multirow{4}{*}{53,3} & Viver & 33,3 \\
\hline & & & & Garantir & 33,3 \\
\hline & & & & Reagir & 6,6 \\
\hline & & & & Autonomizar-se & 6,6 \\
\hline
\end{tabular}

Fonte: dados da pesquisa (2015).

\section{Discussão}

Discute-se, de seguida, os resultados relativos à concepção dos fatores e das funções da aprendizagem observados nos estudantes entrevistados.

Considerando, em primeiro lugar, os resultados relativos à concepção dos fatores de aprendizagem, é possível constatar a dicotomia observada em outros estudos (DUARTE, 2007; RENDEIRO, 2005) entre fatores Ambientais e Pessoais. Esta dicotomia pode indiciar a intuição, nos estudantes, de um princípio de determinismo recíproco, entre organismo e ambiente, para explicar a aprendizagem. O fato de os fatores Pessoais apresentarem maior incidência (presentes em 100\% dos participantes) que os fatores Ambientais pode dever-se ao nível de desenvolvimento e escolaridade dos entrevistados, no qual será natural uma atribuição sobretudo interna das causas da aprendizagem. 
Entre os fatores ambientais, os sociais (meio cultural e social) apresentam maior relevo, o que talvez se explique em função do elevado coletivismo da cultura brasileira, na qual o grupo de pertença e o meio social são importantes fatores do funcionamento psicológico (HOFSTEDE; HOFSTEDE; MINKOV, 2010). Por seu lado, a ênfase secundária, mas não negligenciável, no fator Físico da aprendizagem (26,6\%) confirma as ideias de Gifford (2007) sobre a importância dos espaços físicos do campus, sugerindo que eles afetam o comportamento, o rendimento e o desenvolvimento dos estudantes. A consciência do parâmetro social como fator da aprendizagem é igualmente atestada, ao nível dos fatores Pessoais, pela noção de um fator Interpessoal, embora a este nível o fator mais saliente seja sobretudo o Afetivo (Emoções, Persistência e Interesse) (80\%), o que pode ser explicado pela possível auto-observação, por parte dos participantes, da importância da componente emocional e sobretudo da motivação no seu próprio processo de aprendizagem. $\mathrm{O}$ principal fator pessoal da aprendizagem é, para os entrevistados, o Interesse (46,6\%) - revelador de que o envolvimento afetivo na aprendizagem é tido como o seu motor principal. Na mesma linha, entre os fatores Pessoais Cognitivos, salienta-se o papel conferido à Atenção (40\%), em detrimento do Raciocínio (13,3\%).

Atendendo, em segundo lugar, aos resultados relativos à concepção das funções de aprendizagem, o fato destas serem apontadas, principalmente, como de índole Intrapessoal (86,6\%), parece revelar a consciência de que a aprendizagem serve sobretudo a quem aprende. No entanto, é igualmente significativa a incidência da noção de que a aprendizagem tem uma função Interpessoal (partilhar conhecimento e interagir) (40\%), o que mais uma vez pode-se explicar pelo coletivismo da cultura de referência dos entrevistados atrás referida (HOFSTEDE; HOFSTEDE; MINKOV, 2010). Paralelamente ao destaque conferido à função de Conhecer (46,6\%), revelador da consciência do impacto cognitivo da aprendizagem, a ênfase nas funções de Profissionalizar-se (46\%), de Realizar-se (46\%), de Garantir $(33,3 \%)$ e de Viver (33,3\%), não girando em torno de objetivos acadêmicos específicos e nem de curto prazo, pode ser explicada pelo fato dos estudantes participantes serem do Ensino Superior e, nesse sentido, perspectivarem mais a aprendizagem em termos de ganhos de longo prazo e de desenvolvimento pessoal. Este resultado vai de resto no sentido de estudos anteriores (REBELO, 2005; ROSÁRIO et al., 2006), que observaram que a aprendizagem é concebida pelos estudantes como tendo tanto uma função cognitiva, como de desenvolvimento da autonomia e adaptação pessoal. 


\section{Conclusão}

Apesar das suas limitações relacionadas, sobretudo com a dimensão reduzida da amostra, este estudo consolida a noção da investigação fenomenográfica de que existe uma variação na forma como os estudantes concebem os fatores e as funções da aprendizagem. Na lógica de investigação qualitativa utilizada, não se pretende generalizar os resultados para a população, mas para a teoria.

Atendendo à dificuldade observada, de reflexão por parte dos entrevistados sobre os fatores e as funções da aprendizagem, sugere-se a necessidade de que os estudantes exercitem a capacidade de refletir sobre estas dimensões da aprendizagem. Considerando que as suas concepções sobre estes parâmetros da aprendizagem se encontram provavelmente associadas à forma como eles aprendem, parece indispensável que sejam capazes de refletir sobre elas. Essa reflexão poderá contribuir para que os estudantes regulem a sua aprendizagem de forma mais emancipadora, em alternativa a uma aprendizagem regulada externamente. No mesmo sentido, sugere-se que os contextos educativos promovam essa reflexão junto dos professores. Uma compreensão, pelos professores, das concepções pessoais e das concepções dos estudantes sobre os fatores e as funções da aprendizagem poderá constituir um fator de desenvolvimento do ensino. Sugere-se, ainda, a possibilidade de um tipo de intervenção congruente, ao nível do aconselhamento psico-educacional, pelo psicólogo educacional, no sentido de promover a consciencialização, junto dos seus clientes, da concepção sobre os fatores e funções da aprendizagem.

Como linhas de investigação futuras, sugere-se pesquisas que procurem explorar uma possível transformação da concepção sobre os fatores e as funções de aprendizagem ao longo da escolaridade; investigar a forma como elas se relacionam com outras variáveis, tais como, as abordagens à aprendizagem, a área acadêmica, o sexo, a epistemologia pessoal, o contexto social, a concepção de aprendizagem dos professores, etc.; e, finalmente, testar procedimentos de intervenção sobre as concepções dos fatores e das funções da aprendizagem, que possam contribuir para a consciencialização e eventual mudança dessas concepções e, consequentemente, para o melhoramento do processo e do resultado da aprendizagem. 


\title{
Conceptions of Brazilian university students about the factors and functions of learning
}

\begin{abstract}
This article presents some results from an investigation that aimed to explore the conceptions of learning of a group of Brazilian university students in light of the phenomenographic theory: the results on their conceptions about the factors and functions of learning. Fifteen students from a psychology course were interviewed on the basis of a semi-structured interview script. Their responses, subjected to a content analysis, showed the assignment of adaptive, cognitive, motivational and interpersonal functions to learning, as well as personal and environmental factors. Results support the replication of part of the conceptions on the factors and functions of learning previously revealed by other phenomenographic studies, as well as the emergence of apparently new conceptions.
\end{abstract}

Keywords: Academic learning. Conceptions of learning. Phenomenographic.

\section{Concepciones de estudiantes universitarios brasileños sobre los factores y las funciones del aprendizaje}

\section{Resumen}

En este articulo se presenta parte de los resultados de una investigación que tuvo como objetivo explorar las concepciones de aprendizaje de un grupo de estudiantes universitarios brasileños, a la luz de la perspectiva fenomenográfica: los resultados relativos a sus concepciones sobre los factores y funciones de aprendizaje. Quince estudiantes de un curso de Psicología fueron entrevistados con la ayuda de un guión de una entrevista semiestructurada. Las respuestas, sometidas a un análisis de contenido, demostraron la atribución, al aprendizaje, de funciones adaptativas, cognitivas, motivacionales e interpersonales, asi como de factores personales y ambientales. Los resultados apoyan el hallazgo de una réplica de parte de las concepciones sobre los factores y las funciones de aprendizaje previamente revelados por otros estudios fenomenográficos, asi como la aparición de concepciones aparentemente nuevas.

Palabras clave: Aprendizaje académico. Concepciones de aprendizaje. Fenomenografí. 


\section{Referências}

ASIKAINEN, H. Successful learning and studying in biosciences: exploring how students' conceptions of learning, approaches to learning, motivation and their experiences of the teaching-learning environment are related to study success. 2014. 103f. Tese (Doutorado em Ciências da Educação) - Universidade de Helsinki, Finlândia, 2014.

BAKEMAN, R.; GOTTMAN, J. Observing interaction: an introduction to sequential analysis. London: Cambridge University Press, 1997.

BIGGS, J. Student approaches to learning and studying. Melbourne: ACER, 1987.

. Teaching for desired learning outcomes. In: ENTWISTLE, N. (Org.). Handbook of educational ideas and practices. London: Routledge, 1990. p. 681-93.

. Calidad del aprendizaje universitario. Tradução de P. Manzano. Madrid: Narcea, 2010.

BIGGS, J.; MOORE, P. The process of learning. New York: Prentice Hall, 1993.

DUARTE, A. M. Conceptions of learning and approaches to learning in Portuguese students. Higher Education, Amsterdam, v. 54, n. 6, p. 781-794, 2007. DOI: $10.1007 / \mathrm{s} 10734-006-9023-7$

EKLUND-MYRSKOG, G. Student's conceptions of learning in different educational contexts. Higher Education, Amsterdam,v. 35, p. 299-316, 1998.

ENTWISTLE, N. Contrasting perspectives on learning. In: MARTON, F.; HOUNSELl, D.; ENTWISTLE, N. (Ed.). The experience of learning. Edinburgh: The Scottish Academic Press, 1984. p. 1-18.

FLORES, J. Análises de datos cualitativos: aplicaciones a la investigación educativa. Barcelona: PPV, 1994.

FREIRE, L.; DUARTE, A. M. Concepções de aprendizagem em estudantes universitários brasileiros. Psicologia USP, São Paulo, v. 21, n. 4, p. $875-898,2010$. DOI: $10.1590 / \mathrm{s} 0103-65642010000400012$

FREIRE, P. Educação e mudança. São Paulo: Paz e Terra, 2003.

GIFFORD, R. Environmental psychology: principles and practice. 3rd ed. Colville, WA: Optimal Books, 2007. 
HOFSTEDE, G.; HOFSTEDE, G. J.; MINKOV, M. Cultures and organizations: software of the mind. 3rd ed. New York: McGraw-Hill, 2010.

KHAN, S. Phenomenography: a qualitative research methodology in Bangladesh. International Journal on New Trends in Education \& their Implications (IJONTE), Ankara, v. 5, n. 2, p. 34-43, 2014.

LARA, E. et al. Metas, concepciones educativas y enfoques de aprendizaje del alumnado de ciencias del deporte. Revista Internacional de Medicina y Ciencias de la Actividad Física y del Deporte, Madrid, v. 10, n. 41, p. 14-34, 2011.

LEUNG, C.; WONG, B.; WONG, J. Conceptions of learning in pre-service and in-service early childhood education students and the impact of teaching experience. Australasian Journal of Early Childhood, Watson, v. 38, n. 3, p. 57-67, 2013.

LITTO, F. Aprendizagem profunda e aprendizagem de superfície. Disponível em: <http://www2.uol.com.br/aprendiz/n_colunas/f_litto/id171000.htm>. Acesso em: 22 jul. 2015.

MARTON, F. Phenomenography: describing conceptions of the world around us. Institutional Science, Amsterdam, v. 10, p. 177-200, 1981. DOI: $10.1007 / \mathrm{bf00132516}$

. Describing and improving learning. In: SCHMECK, R.

(Org.).Learning strategies and learning styles. New York: Plenum, 1988. p. 53-82.

MARTON, F.; BOOTH, S. Learning and awareness. Mahwah: Lawrence Erlbaum, 1997.

MARTON, F.; DALL'ALBA, G.; BEATY, E. Conceptions of learning. International Journal of Educational Reserch, Northern Ireland, v. 19, n. 3, p. 277-300, 1993.

MARTON, F.; FAI, P. M. Two faces of variation. In: EUROPEAN CONFERENCE FOR LEARNING AND INSTRUCTION, 8., 1999, Göteborg. Paper... Sweden: Göteborg University, 1999.

MARTON, F.; SÄLJÖ, R. On qualitative differences in learning: I - outcome and process. British Journal of Educational Psychology, v. 46, n. 1, p. 4-11, 1976. DOI: 10.1111/j.2044-8279.1976.tb02980.x 
MARTON, F.; SÄLJÖ, R. Approaches to learning. In: MARTON, F.; HOUNSELL, D.; ENTWISTLE, N. (Org.).The experience of learning. Edinburgh: Scottish Academic Press, 1977. p. 39-58.

PRAMLING, I. The origin of the child's idea of learning through practice. European Journal of Education, 3, 31-46, 1986.

REBELO, M. Concepções de aprendizagem com o computador em estudantes universitários. 2005. Dissertação (Mestrado em Ciências da Educação) - Faculdade de Psicologia e Ciências da Educação. Universidade de Lisboa, Lisboa, 2005.

RENDEIRO, A. Concepções de aprendizagem para a avaliação em estudantes do ensino secundário.2005.Dissertação (Mestrado em Ciências da Educação) - Faculdade de Psicologia e Ciências da Educação. Universidade de Lisboa, Lisboa, 2005.

ROSÁRIO, P. et al. Discursos de pais e alunos sobre o aprender: um estudo no $5^{\circ}$ ano de escolaridade. Psicologia em Estudo, Maringá, v. 11,n. 3, p. 463-471, 2006. DOI: $10.1590 / \mathrm{s} 1413-73722006000300002$

SÄLJÖ, R. Learning from reading. In: MARTON, F.; HOUNSELL, D.; ENTWISTLE, N. (Org.). The experience of learning. Edinburgh: Scottish Academic Press, 1984. p. 71-89.

YANG, Y.-F.; TSAI, C.-C. Conceptions of and approaches to learning through online peer assessment. Learning and Instruction, Oxford, v. 20, n. 1, p. 72-83, 2010. DOI: 10.1016/j.learninstruc.2009.01.003

\section{Informações dos autores}

Luiz Gustavo Lima Freire: Doutorando em Psicologia pela Universidade de Lisboa, Faculdade de Psicologia. Professor da Faculdade dos Guararapes Laureate International Universities - Pernambuco. Contato: luizgustavolfreire@ig.com.br

António Manuel Duarte: Doutor. Professor da Faculdade de Psicologia da Universidade de Lisboa. Contato: amduarte@psicologia.ulisboa.pt 